\title{
Tumor bed radiotherapy in women following breast conserving surgery for breast cancer-safety margin with/without image guidance
}

\author{
ALES HLAVKA ${ }^{1,2}$, JAROSLAV VANASEK ${ }^{1-3}$, KAREL ODRAZKA ${ }^{1,4-6}$, JAN STUK ${ }^{1,2}$, MARTIN DOLEZEL ${ }^{1,4,7}$, \\ VIT ULRYCH $^{1}$, MARTINA VITKOVA $^{1}$, JIRI MYNARIK ${ }^{1}$, IVETA KOLAROVA $^{1-3}$ and ZDENKA VILASOVA ${ }^{1,3}$ \\ ${ }^{1}$ Department of Clinical and Radiation Oncology, Oncology Centre, Multiscan and Pardubice Hospital, 53002 Pardubice; \\ ${ }^{2}$ Faculty of Military Health Sciences, University of Defense, 50001 Hradec Kralove; ${ }^{3}$ Faculty of Health Studies, \\ Pardubice University, 53003 Pardubice; ${ }^{4}$ First Faculty of Medicine and ${ }^{5}$ Third Faculty of Medicine, \\ Charles University, 12108 Prague; ${ }^{6}$ Institute for Postgraduate Medical Education, 10005 Prague; \\ ${ }^{7}$ Faculty of Medicine and Dentistry, Palacký University Olomouc, 77147 Olomouc, Czech Republic
}

Received October 18, 2017; Accepted January 11, 2018

DOI: $10.3892 / \mathrm{ol} .2018 .8083$

\begin{abstract}
Image guided radiation therapy (IGRT) enables the achievement of higher precision in radiation delivery, a reduction in safety margins and a reduced risk of toxicity in healthy tissues. The present study investigated the magnitude of safety margins for the radiation boost setup on skin marks or metal clips implanted into the tumor bed during breast cancer surgery. One hundred eighty-four patients after breast conserving surgery with implanted metal clips into tumor bed were analyzed. The present study investigated the difference in safety margin required for the treatment setup on skin marks and metal clips. The skin marks were created using a positioning laser system in the treatment room. Metal clips implanted in the tumor bed were registered using IGRT with kilovoltage X-rays in orthogonal projection. Treatment setup was performed during free breathing. The safety margin corresponding to the planning target volume (PTV) was calculated from the recorded data. Calculated safety margins for the treatment setup on skin marks were 9.4, 11.1 and $11.1 \mathrm{~mm}$ in the anteroposterior, craniocaudal, and laterolateral directions, respectively. Corresponding safety margins with the use of IGRT and metal clips registration were 4.7, 5.1 and $5.9 \mathrm{~mm}$, respectively. The safe PTV margin was $12 \mathrm{~mm}$ using setup on skin marks without IGRT, whereas a 6-mm margin was sufficient with the use of metal clip-based IGRT with daily online correction. IGRT has been adopted as the standard treatment method within the Oncology Centre of Multiscan and Pardubice Hospital (Pardubice, Czech Republic).
\end{abstract}

Correspondence to: Professor Karel Odrazka, Department of Clinical and Radiation Oncology, Oncology Centre, Multiscan and Pardubice Hospital, 44 Kyjevska, 53002 Pardubice, Czech Republic E-mail: odrazka@seznam.cz

Key words: breast cancer, fiducials, image-guided, radiotherapy

\section{Introduction}

Radiation therapy represents one of the basic treatment modalities in the management of breast cancer. Adjuvant radiotherapy is a standard procedure particularly in women following breast conserving surgery $(1,2)$.

External-beam radiation therapy with conventional fractionation usually includes irradiation of the whole breast followed by a boost dose to the tumor bed. One of the primary challenges of this type of therapy is an accurate focusing on the target volume due to breast mobility and limited options for fixation of the organ. These difficulties are particularly important when using advanced conformal techniques of radiotherapy, primarily intensity modulated radiation therapy (IMRT), which is beneficial for complicated clinical cases, including bilateral breast carcinoma (3).

Utilization of the image guided radiation therapy (IGRT) technique constitutes one of the ways to achieve higher accuracy in target focusing. There are a number of IGRT methods. Currently, the technique that is used most frequently is the kilovoltage X-ray imaging of patients immediately before treatment. The IGRT device is usually integrated into the treatment unit (4).

For the breast area, it is possible to use the X-ray contrast metallic markers that were implanted into the tumor bed during surgery. Such markers may be employed in two ways for accurate localization of the boost volume during treatment planning and subsequently for daily monitoring of the patient position using the selected method of IGRT. Special markers are necessary when the megavoltage beam is used for the treatment setup, while surgical clips are adequate in situations where lower beam intensities are used (5). The implanted clips are typically stable in the tumor bed throughout the course of radiation therapy $(6)$.

The benefit of IGRT is superior in its ability to reduce the safety margin around the target volume, thus decreasing the irradiated breast volume. It may result in lower risks of the radiation fibrosis, and lower doses reaching the lungs 
and heart (in left-sided tumors). Another advantage is more improved coverage of the target volume with the planned dose, which increases the probability of appropriate treatment efficiency (6).

The aim of the present study was to identify the specific values of the safety margins for the irradiation of the tumor bed (boost dose) with and without the clip-based IGRT technique prior to treatment.

\section{Patients and methods}

Patients and treatment. The present study did not result in any interventions related to participating patients. Application of the IGRT technique with registration of the clips in tumor bed is a component of standard treatment, more precisely the target volume localization. The study design enabled a direct comparison of localization with and without IGRT. There were no ethical issues regarding the use of various treatment methods because all patients were treated using IGRT and the setup errors were evaluated based on standard imaging.

The study population included 184 consecutive patients who were treated with radiation for breast cancer at our institution between March 2013 and April 2014. The mean age was 62 years (range, 28-81 years). All patients underwent breast conserving surgery, and had biopsy-verified invasive breast cancer stage I or II, experienced surgical staging of the axillary region (sentinel lymph nodes biopsy or axillary dissection), had metal clips implanted into the tumor bed and were referred to undergo postoperative whole breast radiation with boost dose to the tumor bed. Two women had postoperative radiation of both breasts simultaneously. Written informed consent was obtained from all patients.

Radiation was delivered by the linear accelerator Clinac 2100 C/D (Varian Medical Systems, Inc., Palo Alto, California, USA) using a 3D conformal radiation therapy with photon beams (6 MV and/or $18 \mathrm{MV}$ ). The whole breast was treated with a dose of 48.6, $1.8 \mathrm{~Gy} /$ fraction. Then, the tumor bed was boosted with a dose of 9-12.6, 1.8 Gy/fraction.

Computed tomography (CT) simulation was performed during free breathing on the CT scanner Somatom Definition AS (Siemens AG, Munich, Germany) with following the parameters: Spiral mode, slice thickness $5 \mathrm{~mm}$, slice spacing $5 \mathrm{~mm}$, field of view 50 or $78 \mathrm{~cm}$. Each focus in the space was precisely defined using the Cartesian coordinate system X, Y, $Z$. This data was transferred to the online planning system Eclipse 8.6 (Varian Medical Systems, Inc.). Immobilization was accomplished using the Breastboard fix board with arm and wrist support on the affected side (Civco Rabiotherapy, Inc., Orange City, IA, USA).

The clinical target volume (CTV) was defined as the area of all metal clips and other postoperative changes, while the planning target volume (PTV) was defined as the CTV plus a margin of $1 \mathrm{~cm}$ (automatic 3D expansion) with elimination of the margin $0.5 \mathrm{~cm}$ under the skin surface. Metal markers were delineated as a separate structure. Next, 3D conformal planning was performed using a technique of tangential wedged fields with multileaf collimator shaping. Irradiation was delivered using the linear accelerator Clinac $2100 \mathrm{C} / \mathrm{D}$. The total boost dose to the tumor bed was 9-12.6 Gy, dose per fraction
1.8 Gy, which was preceded by the whole breast irradiation with a dose of $48.6 \mathrm{~Gy}$, dose per fraction $1.8 \mathrm{~Gy}$. Localization of the treatment plan isocenter was verified by checking the position on CT simulator.

\section{Acquisition of image data for IGRT}

2D/2D perpendicular $k V$ imaging. Patient imaging was performed daily before every treatment fraction. Before the boost treatment, patients were positioned on the isocenter skin marks that were painted at simulation. Anteroposterior (AP) and laterolateral (LL) scanning on the $\mathrm{kV}$ (kilovoltage) imaging system of the linear accelerator Clinac $2100 \mathrm{C} / \mathrm{D}$ was used. The images obtained were compared with the AP and LL digitally reconstructed radiographs acquired from the planning CT. Online correction of the patient position was performed by a radiation oncologist prior to the treatment start using metal clips in the breast, thus determining the area of the tumor bed implanted by the surgeon during the course of surgery. Overall, 184 women had 1,042 setup errors measurements, which are the shifts in metal markers in relation to skin marks.

Evaluation of the intra-fraction movement. The position of the metal markers was checked prior to and following treatment in 22 patients. Patient imaging was performed with the AP and LL imaging using the $\mathrm{kV}$ imaging device on the linear accelerator Varian Clinac 2100 C/D. The results of 118 measurements were obtained.

Evaluation of the inter-observer error. The offline check of the metal markers matching was performed by another physician in 20 patients. Localization shifts between the first and second physician were compared and the resulting differences were used for the calculation of the safety margin according to the van Herk formula (7). The results of 103 measurements were obtained.

Breathing excursions. The patients were not instructed to any change of breathing, both the treatment setup and irradiation thus took place during free breathing. In a group of ten patients, the magnitude of the breast movement (metal markers) was monitored. During free breathing, the maximum amplitudes of the marker movement were tracked using 4D CT. The difference in the position of markers was measured at the inspiration/expiration peak.

Quality assurance. An inaccuracy of the presented image in relation to the isocenter of the on-board imaging system on the linear accelerator ( $\mathrm{kV}$ imaging system on the linear accelerator) was stated to be up to $1.5 \mathrm{~mm}$ and the results were monitored regularly. The measurement on the linear accelerator reported that this uncertainty was $<1 \mathrm{~mm}$. The declared tolerance for the treatment table shift uncertainty is $1 \mathrm{~mm}$ in all directions at the most $(8,9)$.

Statistical analysis. Microsoft Excel software (Microsoft Corporation, Redmond, WA, USA) was used to calculate the following parameters: Mean, median, systematic $\left(\sum_{\text {set-up }}\right)$ and random $\left(\sigma_{\text {set-up }}\right)$ error. The safety margins were calculated according to van Herk formula $\left(2.5 \Sigma_{\text {set-up }}+0.7 \sigma_{\text {set-up }}\right)$. 


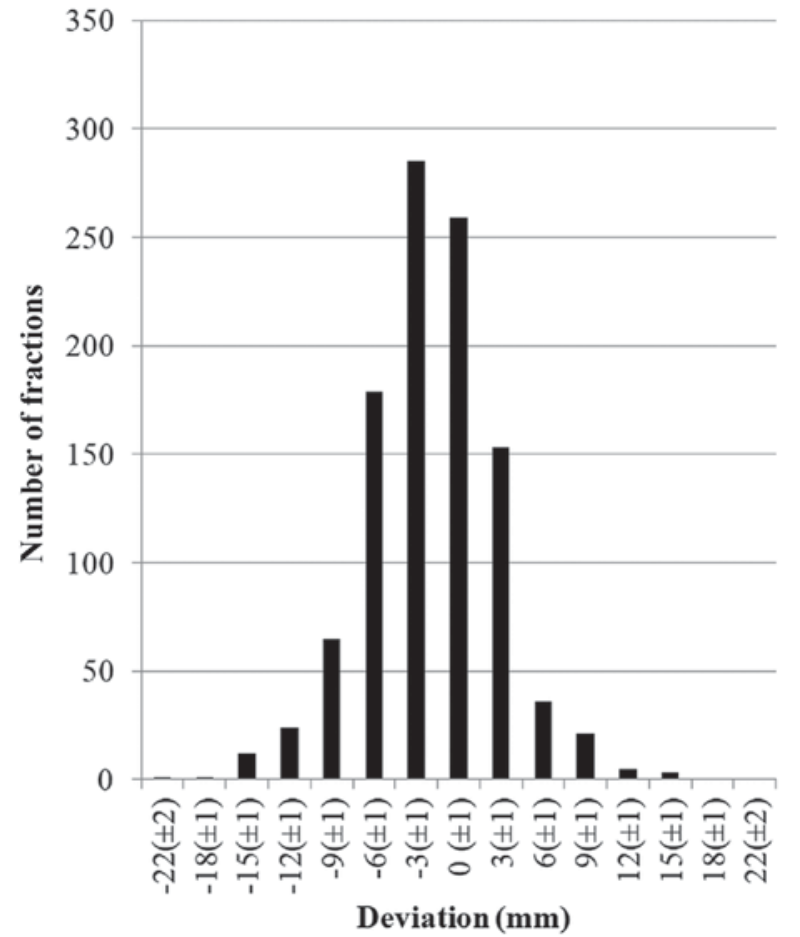

Figure 1. Absolute frequency of setup differences in anterioposterior direction, demonstrating the distribution of differences between setup on skin marks and metal markers in the breast.

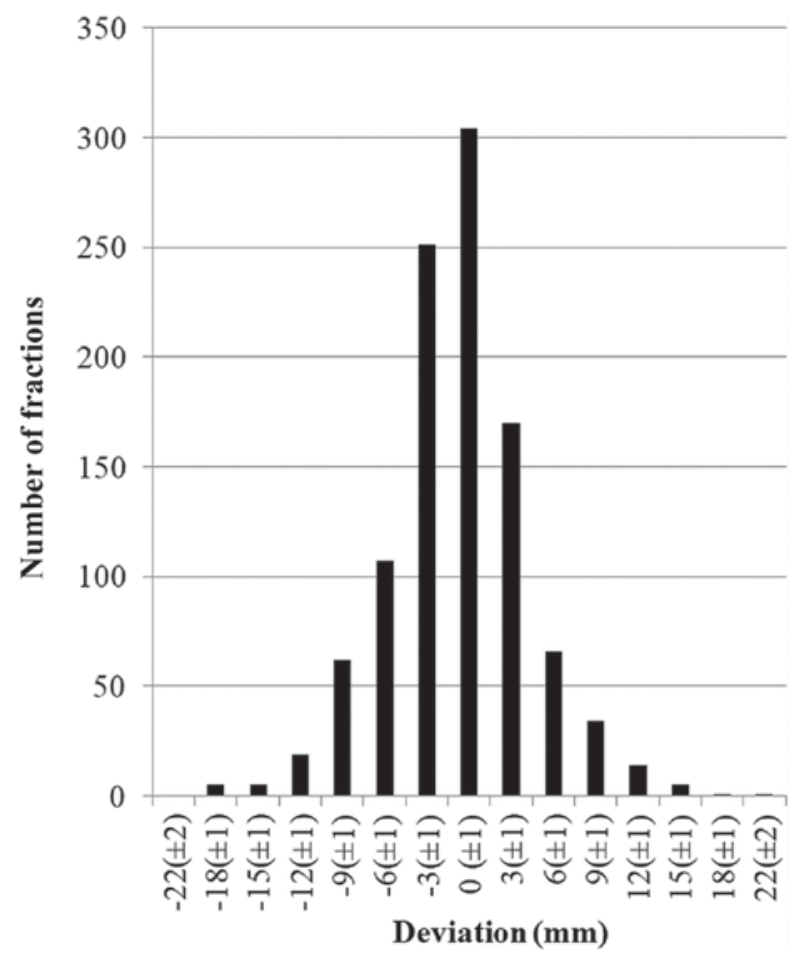

Figure 2. Absolute frequency of setup differences in craniocaudal direction, demonstrating the distribution of differences between setup on skin marks and metal markers in the breast.

The magnitude of the margin between CTV and PTV, calculated according to the aforementioned formula, ensures that $\geq 90 \%$ of the CTV is covered by the $95 \%$ of the dose (7).

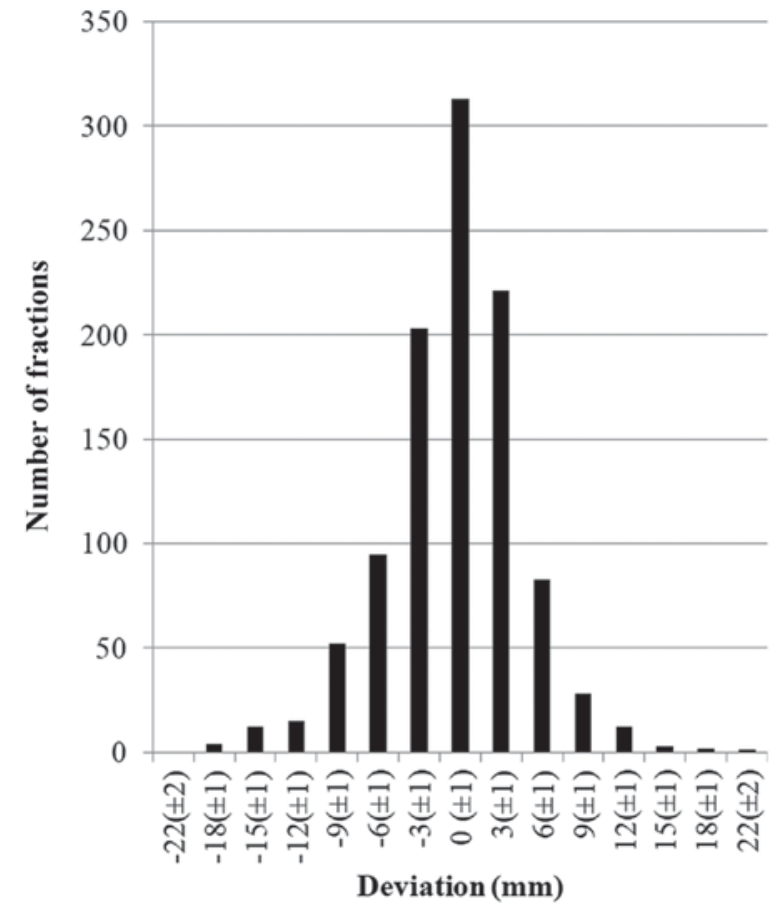

Figure 3. Absolute frequency of setup differences in laterolateral direction, demonstrating the distribution of differences between setup on skin marks and metal markers in the breast.

\section{Results}

Assessment of the required safety margin using the setup on skin marks without IGRT. A total of 1,042 measurements in the group of 184 patients were assessed. Using $\mathrm{kV} 2 \mathrm{D} / 2 \mathrm{D}$ imaging, the difference between setup on skin marks and metal markers in the breast was between- 21 and $+16 \mathrm{~mm}$ in the AP direction (mean, $-2.0 \mathrm{~mm}$ ) (Fig. 1). According to van Herk formula, the calculated margin for the skin mark-based setup was $9.4 \mathrm{~mm}$. In the craniocaudal (CC) (Fig. 2) and LL (Fig. 3) directions, the corresponding values were -18 to $25 \mathrm{~mm}$, mean, $-0.9 \mathrm{~mm}$, margin $11.1 \mathrm{~mm}$; and -19 to $+21 \mathrm{~mm}$, mean $-0.4 \mathrm{~mm}$, margin $11.1 \mathrm{~mm}$, respectively (Table I). Based on this data, the most commonly used margin of $10 \mathrm{~mm}$ between CTV and PTV was insufficient.

A setup error exceeding $10 \mathrm{~mm}$ was observed in $12.3 \%$ of all fractions (128/1,042 fractions). When focused on particular axes, the setup errors exceeding $10 \mathrm{~mm}$ in LL, AP and CC directions were noted in $4.7,4.5$, and $4.8 \%$ of all fractions, respectively.

The setup error exceeding $10 \mathrm{~mm}$ observed in at least one fraction was identified in $40.9 \%$ of patients (76/184 patients). Focusing on this group of 76 patients, the setup error exceeding $10 \mathrm{~mm}$ was registered in $29.5 \%$ of fractions.

The findings from the 4D CT analysis demonstrated that the marker movement in the breast during calm and uncontrolled breathing corresponded to 1-3 $\mathrm{mm}$ in all directions, with $95 \%$ of measurements being $\leq 2.1 \mathrm{~mm}$. The calculated vector magnitude of the marker movement during breathing did not exceed $3.1 \mathrm{~mm}$.

Evaluation of the interobserver error. The results of 103 measurements were acquired when comparing the 
Table I. Differences in setup on skin marks vs. markers in the breast.

\begin{tabular}{lcccccr}
\hline $\begin{array}{c}\text { Maximum } \\
\text { Axis }\end{array}$ & $\begin{array}{c}\text { Maximum } \\
\text { deviation }-(\mathrm{mm})\end{array}$ & $\begin{array}{c}\text { Median } \\
(\mathrm{mm})\end{array}$ & $\begin{array}{c}\text { Mean } \\
(\mathrm{mm})\end{array}$ & $\begin{array}{c}\sigma_{\text {set-up }} \\
\text { deviation }+(\mathrm{mm})\end{array}$ & $\begin{array}{c}\sum_{\text {set-up }} \\
\text { margin }(\mathrm{mm})\end{array}$ \\
\hline AP & -21 & 16 & -2 & -2.0 & 4.2 & 2.6 \\
CC & -18 & 25 & 1 & -0.9 & 4.2 & 3.3 \\
LL & -19 & 21 & 0 & -0.4 & 4.1 & 3.3 \\
\hline
\end{tabular}

AP, anterioposterior; CC, craniocaudal; LL, laterolateral; $\sigma_{\text {set-up }}$, random error; $\Sigma_{\text {set-up }}$, systematic error.

Table II. Interobserver differences in setup on markers in the breast.

\begin{tabular}{lccccccc}
\hline Axis & $\begin{array}{c}\text { Maximum } \\
\text { deviation }-(\mathrm{mm})\end{array}$ & $\begin{array}{c}\text { Maximum } \\
\text { deviation }+(\mathrm{mm})\end{array}$ & $\begin{array}{c}\text { Median } \\
(\mathrm{mm})\end{array}$ & $\begin{array}{c}\text { Mean } \\
(\mathrm{mm})\end{array}$ & $\begin{array}{c}\sigma_{\text {set-up }} \\
\sum_{\text {set-up }}\end{array}$ margin $(\mathrm{mm})$ \\
\hline AP & -1 & 3 & 0 & 0.1 & 0.6 & 0.3 & 1.1 \\
CC & -2 & 2 & 0 & -0.1 & 0.7 & 0.3 & 1.2 \\
LL & -3 & 2 & 0 & -0.1 & 0.6 & 0.3 & 1.2 \\
\hline
\end{tabular}

AP, anterioposterior; CC, craniocaudal; LL, laterolateral; $\sigma_{\text {set-up }}$, random error; $\Sigma_{\text {set-up }}$, systematic error.

Table III. Intrafraction differences in setup on markers in the breast.

\begin{tabular}{|c|c|c|c|c|c|c|c|}
\hline Axis & $\begin{array}{c}\text { Maximum } \\
\text { deviation - }(\mathrm{mm})\end{array}$ & $\begin{array}{c}\text { Maximum } \\
\text { deviation }+(\mathrm{mm})\end{array}$ & $\begin{array}{l}\text { Median } \\
(\mathrm{mm})\end{array}$ & $\begin{array}{l}\text { Mean } \\
(\mathrm{mm})\end{array}$ & $\sigma_{\text {set-up }}$ & $\sum_{\text {set-up }}$ & $\begin{array}{l}\text { Calculated } \\
\text { margin }(\mathrm{mm})\end{array}$ \\
\hline AP & -5 & 4 & -1 & 1.2 & 1.6 & 1.2 & 4.1 \\
\hline $\mathrm{CC}$ & -7 & 4 & -1 & -1.2 & 1.6 & 1.4 & 4.5 \\
\hline LL & -9 & 5 & 0 & -0.5 & 1.9 & 1.6 & 5.4 \\
\hline
\end{tabular}

AP, anterioposterior; CC, craniocaudal; LL, laterolateral; $\sigma_{\text {set-up }}$, random error; $\Sigma_{\text {set-up }}$, systematic error.

interobserver differences in setup correction. The maximum differences in the AP, CC, and LL directions were -1 to $+3 \mathrm{~mm},-2$ to $+2 \mathrm{~mm}$, and -3 to $+2 \mathrm{~mm}$, respectively. The corresponding values of the calculated safety margin were 1.1, 1.2 and $1.2 \mathrm{~mm}$, respectively (Table II).

Evaluation of the intrafraction movement. The results of 118 measurements were obtained during the comparison of the intrafraction movement. The intrafraction movement, which is the difference in $\mathrm{kV} 2 \mathrm{D} / 2 \mathrm{D}$ imaging between setup on the metal markers prior to and following irradiation, was -5 to $+4 \mathrm{~mm},-7$ to $+4 \mathrm{~mm}$, and -9 to $+5 \mathrm{~mm}$ in the AP, CC, and LL directions, respectively. The corresponding values of the safety margin calculated according to the van Herk formula were 4.1, 4.5 and $5.4 \mathrm{~mm}$, respectively (Table III).

Margin calculations using IGRT. The PTV margin for daily online verification of the marker position in the breast was calculated. The intrafraction movement (IF), interobserver differences in setup correction (IO) and respiratory induced movement (RM) of the markers during free breathing were included in the calculation. The total margin was calculated as a standard deviation as follows: $\sqrt{\mathrm{IF}^{2}+\mathrm{IO}^{2}+\mathrm{RM}^{2}}$. The resulting margins in AP, CC and LL directions were 4.7, 5.1, and $5.9 \mathrm{~mm}$, respectively (Table IV) (7).

\section{Discussion}

The aim of the present study was to assess the appropriateness of IGRT technique use, based on the monitoring of the marker position in the area of tumor bed following surgical removal of tumors. As is evident from the literature, the implanted clips are typically stable in the tumor bed throughout the six-week course of radiation therapy (6). Park et al (10) investigated the locations of the clips prior to and following accelerated partial breast irradiation in 26 patients using 4D CT, and observed position changes $\leq 1 \mathrm{~mm}$ (10). Weed et al (11) monitored the changes in clip locations and resection cavity size in 28 patients with two CT scans at a mean of 27 days apart. A mean shift of $3 \mathrm{~mm}$ was reported in all three axes with a decreasing volume in the resection cavity (11). In the present cohort, no significant movement of the clips was observed. Nevertheless, tracking of the clips was limited to a period of 6 to 9 days, which was the time between the $\mathrm{CT}$ for boost planning and 
Table IV. Calculated planning target volume margins for daily online verification of the marker position in the breast.

\begin{tabular}{lcccc}
\hline Axis & IF (mm) & IO (mm) & RM (mm) & $\begin{array}{c}\text { Calculated } \\
\text { margin }(\mathrm{mm})\end{array}$ \\
\hline AP & 4.1 & 1.1 & 2.1 & 4.7 \\
CC & 4.5 & 1.2 & 2.1 & 5.1 \\
LL & 5.4 & 1.2 & 2.1 & 5.9 \\
\hline
\end{tabular}

AP, anterioposterior; CC, craniocaudal; LL, laterolateral; IF, intrafraction movement; IO, interobserver differences in setup correction; $\mathrm{RM}$, respiratory induced movement.

the last fraction of radiotherapy. Observation was realized a long time following clip implantation and so the changes in resection cavity were minor. Thus, the clips are suitable for position monitoring. More accurate localization of the target volume enabled a reduction in the magnitude of the safety margin, thus decreasing the dose delivered to the surrounding healthy tissues. Another advantage is that precise localization limits the possibility of a geographical miss, which may result in target volume underdosage. This is primarily important when using the boost dose (6).

Marker movement in the breast during free breathing, which was observed in the present study, corresponds with published data. Wang et al (12) performed 4D CT in 17 patients and reported a motion vector of $2.09 \pm 0.94 \mathrm{~mm}$. Similarly, Richter et al (13) reported a motion vector of $1.8 \pm 0.9 \mathrm{~mm}$ using the 4D CT in a group of 10 patients.

Numerous studies were performed examining the proper magnitude of safety margin when the setup was guided by skin marks. Yue et al (14) investigated setup errors in 21 patients using three types of localization: Setup on skin marks, daily localization with $\mathrm{kV}$ portal images of the anatomic bony structures, and localization of markers implanted into the tumor bed. The mean interfraction setup error was $9 \mathrm{~mm}$ for skin marks and $7.1 \mathrm{~mm}$ for bony structures in relation to metal markers (14).

Hasan et al (15) compared the registration of the simulation CT with subsequent CT, and analyzed the position of bony structures, clips and breast surface. It was demonstrated that following partial mastectomy the cavity localization using setup on bony structures was the worst, while the localization using clips was very stable in relation to anatomic changes (15). The importance of surgical clips for the IGRT localization was investigated by Topolnjak et al (16). The authors used cone beam CT in 21 patients and retrospectively compared the position of clips in relation to the cavity following surgery. During treatment, the mean difference between the clip positions in relation to the cavity center was $1.4 \mathrm{~mm}$, with a maximum distance of $5.8 \mathrm{~mm}$. The authors concluded that surgical clips are useful for the localization of the cavity following tumor excision. Harris et al (17) evaluated position of the markers implanted into the excision cavity using daily portal imaging and a CT at the end of treatment. The maximum change of the position prior to and following treatment was $7 \mathrm{~mm}$ (17). Coles et al (18) organized a prospective study in 42 evaluable patients to identify the required safety margin when using gold seeds implanted into the tumor bed. CT monitoring of the seed position has been used. An analysis revealed that a margin of $10.1 \mathrm{~mm}$ was necessary when no correction was performed (setup according to skin marks), whereas a margin of $1.4 \mathrm{~mm}$ was sufficient with the correction protocol based on daily localization (18).

Using 4D CT imaging, Latifi et al (6) evaluated migration of markers during the 6-week course of radiation therapy. Only minimal positional changes have been observed. The authors also investigated the impact of respiratory movements on the position of markers and seroma, intrafraction, and interfraction. Such positional uncertainties were $<1 \mathrm{~mm}$ (mean value). According to results of the aforementioned study, a PTV margin of $7 \mathrm{~mm}$ was insufficient in the absence of the marker-based IGRT. A safety margin of at least $9 \mathrm{~mm}$ is necessary for the daily setup using bony structures. In order to achieve more precise positioning, daily localization of the clips using IGRT appears to be essential. In this case, the PTV safety margin may be limited to $6 \mathrm{~mm}(6)$. In 2014, Harris et al (19) published the results of a British multicenter study investigating the safety margin magnitude in 60 patients with boost irradiation (sequential boost in 30 patients, simultaneous integrated boost in 30 patients). Two sizes of the safety margin were compared, 5 vs. $8 \mathrm{~mm}$. The authors concluded that a margin of $5 \mathrm{~mm}$ was sufficient when IGRT localization of the surgical clips was utilized (19).

In conclusion, the results of the present study suggest that the commonly used PTV margin of $10 \mathrm{~mm}$ is insufficient for the setup on skin marks (calculated margin in the present cohort was $12 \mathrm{~mm}$ ). It is possible to reduce the safety margin $<10 \mathrm{~mm}$ using IGRT localization of the metal markers in the breast (calculated margin in the present cohort was $6 \mathrm{~mm}$ ). The resulting size of the calculated safety margin depends on the technical equipment and staff quality of a particular department.

\section{Acknowledgements}

The authors would like to thank Mrs. Lenka Jezkova (Oncology Centre, Multiscan Pardubice) for help with checking the text and citations.

\section{Funding}

No funding was received.

\section{Availability of data and materials}

The datasets used and/or analyzed during the current study are available from the corresponding author on reasonable request

\section{Authors' contributions}

AH, JV and KO designed the study. AH, KO, JS, MD, VU, MV and IK collected the data. ZV digitalized the data. JM performed statistical data processing. AH, JV and KO wrote the study. KO made language corrections. 


\section{Ethics approval and consent to participate}

There were no ethical issues regarding the use of various treatment methods because all patients were treated using IGRT and the setup errors were evaluated based on standard imaging. Written informed consent was obtained from all patients.

\section{Consent for publication}

Consent for data to be published is included in the written informed consent from all patients.

\section{Competing interests}

The authors declare that they have no competing interests.

\section{References}

1. Early Breast Cancer Trialists' Collaborative Group (EBCTCG), Darby S, McGale P, Correa C, Taylor C, Arriagada R, Clarke M, Cutter D, Davies C, Ewertz M, et al: Effect of radiotherapy after breast-conserving surgery on 10-year recurrence and 15-year breast cancer death: Meta-analysis of individual patient data for 10,801 women in 17 randomised trials. Lancet 378: 1707-1716, 2011.

2. Fisher B, Anderson S, Bryant J, Margolese RG, Deutsch M, Fisher ER, Jeong JH and Wolmark N: Twenty-year follow-up of a randomized trial comparing total mastectomy, lumpectomy and lumpectomy plus irradiation for the treatment of invasive breast cancer. N Engl J Med 347: 1233-1241, 2002.

3. Fiorentino A, Tebano U, Ruggieri R, Ricchetti F and Alongi F: Simultaneous integrated bilateral breast and nodal irradiation with volumetric arc therapy: Case report and literature review. Tumori J 102 (Suppl 2): S32-S34, 2016.

4. Vanasek J, Odrazka K, Dolezel M, Dusek L, Jarkovsky J, Hlavka A, Valentova E and Kolarova I: Searching for an appropriate image-guided radiotherapy method in prostate cancer-implications for safety margin. Tumori 100: 518-523, 2014.

5. Shaikh T, Chen T, Khan A, Yue NJ, Kearney T, Cohler A, Haffty BG and Goyal S: Improvement in interobserver accuracy in delineation of the lumpectomy cavity using fiducial markers. Int J Radiat Oncol 78: 1127-1134, 2010.

6. Latifi K, Pritz J, Zhang GG, Moros EG and Harris EER: Fiducial-based image-guided radiotherapy for whole breast irradiation. J Radiat Oncol 2: 185-190, 2013.
7. van Herk M, Remeijer P, Rasch C and Lebesque JV: The probability of correct target dosage: Dose-population histograms for deriving treatment margins in radiotherapy. Int J Radiat Oncol Biol Phys 47: 1121-1135, 2000.

8. Varian Medical System: OBI v1.6 and CBCT v2.1 installation product acceptance, 2015.

9. Varian Medical System: High energy c-series clinac installation product acceptance, 2016

10. Park CK, Pritz J, Zhang GG, Forster KM and Harris EE: Validating fiducial markers for image-guided radiation therapy for accelerated partial breast irradiation in early-stage breast cancer. Int J Radiat Oncol Biol Phys 82: e425-e431, 2012.

11. Weed DW, Yan D, Martinez AA, Vicini FA, Wilkinson TJ and Wong J: The validity of surgical clips as a radiographic surrogate for the lumpectomy cavity in image-guided accelerated partial breast irradiation. Int J Radiat Oncol Biol Phys 60: 484-492, 2004.

12. Wang W, Li JB, Hu HG, Li FX, Xu M, Sun T and Lu J: Correlation between target motion and the dosimetric variance of breast and organ at risk during whole breast radiotherapy using 4DCT. Radiat Oncol 8: 111, 2013

13. Richter A, Sweeney R, Baier K, Flentje M and Guckenberger M: Effect of breathing motion in radiotherapy of breast cancer: 4D dose calculation and motion tracking via EPID. Strahlenther Onkol 185: 425-430, 2009.

14. Yue NJ, Goyal S, Zhou J, Khan AJ and Haffty BG: Intrafractional target motions and uncertainties of treatment setup reference systems in accelerated partial breast irradiation. Int J Radiat Oncol Biol Phys 79: 1549-1556, 2011.

15. Hasan Y, Kim L, Martinez A, Vicini F and Yan D: Image guidance in external beam accelerated partial breast irradiation: Comparison of surrogates for the lumpectomy cavity. Int J Radiat Oncol Biol Phys 70: 619-625, 2008.

16. Topolnjak R, de Ruiter P, Remeijer P, van Vliet-Vroegindeweij C, Rasch C and Sonke JJ: Image-guided radiotherapy for breast cancer patients: Surgical clips as surrogate for breast excision cavity. Int J Radiat Oncol Biol Phys 81: e187-e195, 2011.

17. Harris EJ, Donovan EM, Yarnold JR, Coles CE and Evans PM; IMPORT Trial Management Group: Characterization of target volume changes during breast radiotherapy using implanted fiducial markers and portal imaging. Int J Radiat Oncol Biol Phys 73: 958-966, 2009.

18. Coles CE, Harris EJ, Donovan EM, Bliss P, Evans PM, Fairfoul J, Mackenzie C, Rawlings C, Syndikus I, Twyman N, et al: Evaluation of implanted gold seeds for breast radiotherapy planning and on treatment verification: A feasibility study on behalf of the IMPORT trialists. Radiother Oncol 100: 276-281, 2011.

19. Harris EJ, Mukesh M, Jena R, Baker A, Bartelink H, Brooks C, Dean J, Donovan EM, Collette S, Eagle S, et al: A multicentre observational study evaluating image-guided radiotherapy for more accurate partial-breast intensity-modulated radiotherapy: Comparison with standard imaging technique. NIHR Journals Library, Southampton (UK), 2014. 